Empfehlungen der Deutschen Vereinigung zur Bekämpfung der Viruskrankheiten (DVV) e.V. und der Gesellschaft für Virologie (GfV) e.V.

Holger F. Rabenau', René Gottschalk ${ }^{2}$, Lutz Gürtler ${ }^{3}$, Annette E. Haberl ${ }^{4}$, Osamah Hamouda ${ }^{5}$, Heiko Himmelreich ${ }^{6}$, Klaus Korn ${ }^{7}$, Thomas Mertens ${ }^{8}$, Kurt W. Schmidt ${ }^{9}$, Stefan Schmiedel ${ }^{10}$, Andreas Spickhoff ${ }^{11}$, Gaby Wirz $^{12}$, Peter Wutzler ${ }^{13}$, Sabine Wicker ${ }^{14}$

1 Institut für Medizinische Virologie, Klinikum der Johann Wolfgang Goethe-Universität Frankfurt, Frankfurt am Main

2 Amt für Gesundheit, Frankfurt am Main

3 Gräfelfing

4 Medizinische Klinik II; Schwerpunkt Infektiologie HIVCENTER, Klinikum der Johann Wolfgang Goethe-Universität Frankfurt, Frankfurt am Main, Deutschland

5 Fachgebiet HIV/AIDS und andere sexuell oder durch Blut übertragbare Infektionen, Abt. für Infektionsepidemiologie, Robert Koch-Institut, Berlin (Weißensee)

6 Klinik für Unfall-, Hand- und Wiederherstellungschirurgie, Klinikum der Johann Wolfgang Goethe-Universität Frankfurt, Frankfurt am Main

7 Institut für Klinische und Molekulare Virologie, Erlangen

8 Abteilung Virologie, Universitätsklinikum UIm, UIm

9 Zentrum für Ethik in der Medizin, AGAPLESION Markus Krankenhaus, Frankfurt am Main

10 Medizinische Klinik I (Gastroenterologie, Hepatologie, Infektiologie, Tropenmedizin), Ambulanzzentrum Infektiologie \& Bernhard-Nocht-Klinik für Tropenmedizin, Universitätsklinikum Hamburg Eppendorf, Hamburg

11 Bürgerliches Recht, Medizinrecht, IPR und Rechtsvergleichung, Georg-August-Universität Göttingen, Göttingen

12 AIDS-Hilfe Baden-Württemberg e. V., Kornwestheim

13 Institut für Virologie und Antivirale Therapie, Universitätsklinikum Jena, Jena

14 Betriebsärztlicher Dienst, Klinikum der Johann Wolfgang Goethe-Universität Frankfurt, Frankfurt am Main

\section{Prävention der nosokomialen} Übertragung von humanem Immunschwächevirus (HIV) durch HIV-positive Mitarbeiterinnen und Mitarbeiter im Gesundheitswesen

\section{Prevention of nosocomial transmission of human immunode- ficiency virus (HIV) from HIV-positive healthcare workers}

\section{Zusammenfassung}

Die Deutsche Vereinigung zur Bekämpfung der Viruskrankheiten (DVV) e.V. und die Gesellschaft für Virologie (GfV) e.V. geben nach unserem Wissen erstmalig in Europa konkrete Empfehlungen zum Einsatz von HIV-positiven Mitarbeiterinnen und Mitarbeitern im Gesundheitswesen. Grundsätzlich gelten für HIV-positives medizinisches Personal klar definierte Auflagen. Bei einer dauerhaften Viruslast von $\leq 50$ Kopien/ml können unter der Einhaltung der festgelegten Maßnahmen (z. B. Tragen doppelter Handschuhe, regelmäßige arbeitsmedizinische Betreuung, mindestens vierteljährliche Kontrollen der HI-Viruslast, regelmäßige Betreuung durch einen in der HIV-Therapie erfahrenen Arzt) von dem HIV-positiven medizinischen medizinischen Beschäftigten ist jedoch nur dann möglich, wenn Adhärenz bei der antiretroviralen Therapie besteht und wenn alle zur Vermeidung einer Infektionsübertragung erforderlichen Maßnahmen regelrecht eingehalten werden. Unter Einhaltung der Vorgaben dieser Empfehlungen gehen von dem HIV-positiven medizinischen Beschäftigten mit an Sicherheit grenzender Wahrscheinlichkeit keine Gefahren für Patienten aus.

\section{Summary}

To the best of our knowledge, the German Association for the Control of Viral Diseases
Empfehlung

Schlüsselwörter

Infiziertes medizinisches

Personal

Nosokomiale HIV-Infektion

Mitarbeiter im Gesundheitswesen

Transmissionsrisiko

Übertragungsträchtige Tätigkeit

Keywords

Infected health care personnel

Nosocomial HIV infection

Health care workers

Risk of transmission

Exposure-prone procedures

*Korrespondierende Autoren

Prof. Dr. Holger F. Rabenau

Institut für Medizinische Virologie, Klinikum der Johann Wolfgang Goethe-Universität Frankfurt/Main Paul-Ehrlich-Str. 40

60596 Frankfurt/Main

E-Mail:

Rabenau@em.uni-frankfurt.de
PD Dr. Dr. Sabine Wicker

Betriebsärztlicher Dienst

Klinikum der Johann Wolfgang

Goethe-Universität Frankfurt

Theodor-Stern-Kai 7

60590 Frankfurt am Main

E-Mail: Sabine.Wicker@kgu.de

Die Empfehlung ist ein Nachdruck

des Originalbeitrags aus der Zeitschrift Bundesgesundheitsblatt des SpringerVerlags Heidelberg.

Bundesgesundheitsblatt Gesundheitsforschung Gesundheitsschutz. 2012 Aug;55(8):937-43. 
(DVV) e.V. and the Society for Virology (GfV) e.V. are the first in Europe to provide precise recommendations for the management of health care workers (HCWs) who are infected with human immunodeficiency virus (HIV). Requirements for HIV-infected HCWs need to be clearly defined. With a permanent viral burden of less than or equal to 50 copies/mL, HIV-positive HCWs are allowed to perform any surgery and any invasive procedure, as long as the infected HCW uses double-gloving, undergoes follow-up routinely by occupational medicine professionals, undergoes a quarterly examination of viral burden, and has a regular medical examination by a physician who has expertise in the management of HIV. Unrestricted professional activity is only possible with a strict compliance to take antiretroviral therapy and if the HIV-infected HCW strictly adheres to the recommended infection control procedures. Complete compliance with the recommendation almost certainly leads to no HIV transmission risk in patient care.

\section{Einleitung}

Ziel dieser Empfehlung ist die Prävention nosokomialer HIV-Infektionen durch HIVpositive Mitarbeiterinnen und Mitarbeiter im Gesundheitswesen (HCW ${ }^{1}$ ). Die Empfehlung dient primär dem Patientenschutz. Darüber hinaus sollen auch die beruflichen und sozialen Interessen HIV-positiver HCW angemessen berücksichtigt werden.

Die Bewertung der Infektiosität von HIV-positiven HCW sowie die Beurteilung der von ihnen durchführbaren operativen und invasiven Tätigkeiten erfordern fachlich fundierte Einschätzungen. Die vorliegende Empfehlung erstreckt sich auf die im Gesundheitswesen tätigen Personen (Selbstständige und Nicht-Selbstständige) sowohl in der ambulanten als auch in der stationären Versorgung.

Nosokomiale Übertragungen von HIV durch HCW wurden vereinzelt in der internationalen Literatur beschrieben. In Deutschland ist bisher keine solche Transmission dokumentiert worden.

Da die HIV-Übertragung entweder parenteral oder über die Schleimhaut bzw. nichtintakte Haut erfolgt, ist eine HIV-

1 Im Text wird für „Mitarbeiterinnen und Mitarbeiter im Gesundheitswesen“ die englischsprachige Abkürzung für healthcare worker „HCW“ verwendet. Aus Gründen der besseren Lesbarkeit wird auf die gleichzeitige Verwendung männlicher und weiblicher Sprachformen verzichtet. Sämtliche Personenbezeichnungen gelten gleichwohl für beide Geschlechter.
Transmission vom infizierten HCW auf Patienten bei nichtinvasiven medizinischen Versorgungsmaßnahmen mit an Sicherheit grenzender Wahrscheinlichkeit nicht möglich. Auch bei invasiven Maßnahmen ist das Übertragungsrisiko bei Einhaltung krankenhaushygienischer Schutzmaßnahmen als sehr gering einzustufen. Daher können auch im Falle einer HIV-Infektion eines HCW - analog der Empfehlung der DVV zur „Prävention der nosokomialen Übertragung des Hepatitis-B-Virus (HBV) und Hepatitis-C-Virus (HCV) durch im Gesundheitswesen Tätige ${ }^{\star}$ [1] - alle operativen und invasiven Tätigkeiten durchgeführt werden, sofern die HIV-Menge im Blut des betroffenen HCW dauerhaft auf $\leq 50 \mathrm{Kopien} / \mathrm{ml}$ abgesenkt ist und die nachfolgend aufgeführten allgemeinen und verhaltens-präventiven Maßnahmen konsequent beachtet werden. Unter diesen Bedingungen ist eine HIV-Übertragung mit an Sicherheit grenzender Wahrscheinlichkeit nicht möglich. Die uneingeschränkte Tätigkeit des HIV-positiven HCW ist allerdings nur dann möglich, wenn die antiretrovirale Therapie regelmäßig erfolgt und wenn alle zur Vermeidung einer Infektionsübertragung erforderlichen Maßnahmen regelrecht eingehalten werden. Ferner ist ein vertrauensvolles Verhältnis zwischen Betriebsarzt und HCW hierbei Grundvoraussetzung.

Die hier vorgestellten Aspekte zur Prävention einer HIV-Übertragung durch HIVpositive HCW basieren auf 3 Stufen:

- der Festlegung des Tätigkeitsspektrums in Abhängigkeit von der Viruslast,

- den zu erfüllenden Voraussetzungen zum Tätigwerden und

- den zu ergreifenden Schutzmaßnahmen.

\section{Epidemiologische Hintergründe}

Die Übertragung von HIV von einem HIVpositiven HCW auf Patienten ist nach dem aktuellen Stand des Wissens und den epidemiologischen Daten ein sehr seltenes Ereignis. Insgesamt sind bislang nur 4 dokumentierte Kasuistiken von nosokomialen HIV-Übertragungen beschrieben worden:
1. HIV-positiver, symptomatischer Zahnarzt aus den USA, der 19906 von ihm betreute Patienten infizierte (Beweis: $\mathrm{Nu}-$ kleinsäure-Sequenzierung; insgesamt 1100 Look-back-Untersuchungen; [2]). Die Umstände der Übertragung (Absicht, Unfall?) konnten nicht geklärt werden.

2. HIV-positiver, chirurgisch tätiger Orthopäde aus Frankreich, der 1 Patienten infizierte (Beweis: Nukleinsäure-Sequenzierung; 983 Look-back-Untersuchungen). Dem Orthopäden war - zum Zeitpunkt der Übertragung - seine eigene Infektion nicht bekannt [3].

3. HIV-positive Krankenschwester aus Frankreich, die 1 Patientin infizierte (Beweis: Nukleinsäure-Sequenzierung; 2293 Look-back-Untersuchungen; [4]). Der Krankenschwester war - zum Zeitpunkt der Übertragung - die eigene Infektion nicht bekannt. Die Umstände der Übertragung konnten nicht geklärt werden.

4. HIV-positiver Gynäkologe aus Spanien, der 1 Patientin während einer Sectio infizierte (Beweis: Nukleinsäure-Sequenzierung; 250 Look-back-Untersuchungen; [5]).

Inwieweit diese 4 Fälle die tatsächliche Transmissionshäufigkeit widerspiegeln oder aufgrund von Nicht-Erkennung respektive Nicht-Publikation die Infektionsübertragungszahlen höher liegen, ist letztendlich nicht geklärt.

Auf der Basis von Vergleichen mit der Übertragbarkeit von HBV und HCV im Rahmen von Nadelstichverletzungen (NSV) ist bekannt, dass HIV seltener übertragen wird. Das HIV-Übertragungsrisiko wird nach perkutaner Exposition mit blutkontaminierten scharfen Instrumenten mit $0,32 \%$ angegeben [21 nachweisliche Infektionen, die mit 6498 Expositionen assoziiert wurden (95\%-Konfidenzintervall: 0,18-0,46\%); [6]].

In 2 Studien konnte gezeigt werden, dass es bei intraoperativen Verletzungen von HCW in 11,4-29 \% zu einem erneuten Kontakt des verletzenden Instruments mit dem Patienten kam [7, 8]. Bei Schleimhautexposition liegt das Risiko etwa um den Faktor 10 niedriger und wird mit 0,03\% angegeben (2 nachweisliche Infektionen, die mit 2886 Expositionen assoziiert wurden; $[9,10])$. Bei verschiedenen Look-backUntersuchungen an 22.171 Patienten (entspricht jedoch lediglich ca. $17 \%$ der betreuten Patienten), die von 51 HIV-positiven HCW betreut wurden, konnten keine 
Übertragungen auf Patienten dokumentiert werden [11].

Hochrechnungen weisen darauf hin, dass in Deutschland über 5000 HIV-positive Mitarbeiter im Gesundheitswesen tätig sind [12]. Dieser Schätzung zugrunde gelegt wird eine Gesamtbevölkerungszahl von 82 Mio. mit 43,5 Mio. Erwerbstätigen und 4,7 Mio. im Gesundheitswesen Tätigen (etwa jeder 9. Beschäftigte; [13]). Derzeit leben in Deutschland ca. 73.000 HIVInfizierte [14], von denen schätzungsweise etwa zwei Drittel täglich zur Arbeit gehen [15]; daraus resultieren ca. 48.000 HIV-positive Berufstätige und ca. 5300 Menschen mit HIV, die im Gesundheitswesen tätig sind.

\section{Biologische Grundlagen zur Risikoabschätzung einer HIV-Übertragung}

Bei der Replikation von Viren treten grundsätzlich neben infektiösen Virusnachkommen auch solche auf, die replikationsdefekt und damit nicht mehr infektiös sind. Verschiedene In-vitro-Untersuchungen weisen darauf hin, dass auf ein infektiöses HI-Virus, abhängig vom Zellkultursystem, zwischen 250 und 50.000 Viruspartikel (d. h. 500 bis 100.000 RNA-Kopien) kommen, die im Zellkultursystem keine Infektiosität aufweisen [16].

In vivo kann HIV frei im Plasma oder zellgebunden vorhanden sein. Die humane infektiöse Dosis (HID), die zur Übertragung auf den Menschen notwendig ist, kann aus In-vitro-Daten sowie indirekt aus Infektionsereignissen nach Stichverletzungen berechnet werden. Nach letztgenannten Berechnungen beträgt die HID zwischen 100 und 1000 Viruspartikel, im Mittel wahrscheinlich 300 bis 500 Viruspartikel. Grundlage der Kalkulation sind oberflächliche Kanülenverletzung und Spritzer ins Auge [10, 17]. Ein ähnlicher Wert ergibt sich aus Übertragungsexperimenten von in Zellkulturen gezüchtetem HIV auf Schimpansen [18].

Prinzipiell steigt die Übertragungswahrscheinlichkeit proportional zur übertragenen Blutmenge [19], mit der Höhe der Viruslast (HIV-RNA-Kopien/ml) des Indexpatienten und der Dauer, die das eingebrachte Material im Stichkanal oder auf der Schleimhaut verbleibt.

Neben den viralen Faktoren (Zelltropismus, Menge infizierter Zellen) sind auch genetische Faktoren des Menschen für die Infektionswahrscheinlichkeit bedeutend (z. B. genetische Defekte wie CCR5 $\triangle 32$ ).

\section{Klinische Bewertung der Infektiosität}

Durch Suppression der Virusreplikation mittels antiretroviraler Kombinationstherapie (ART) kann die Virusmenge im Blut dauerhaft bis unter die Nachweisgrenzeetwa 20 bis 50 Viruskopien/ml Blut - gesenkt werden. In der Folge kommt es zu einer Verbesserung bzw. Stabilisierung des Immunsystems. Wird die HIV-Therapie rechtzeitig begonnen, haben HIV-Positive eine annähernd normale Lebenserwartung [20] und darüber hinaus meist die Möglichkeit einer normalen Teilhabe am Berufsleben. Zudem weisen Studienergebnisse darauf hin, dass eine gut eingestellte HIVTherapie auch die Infektiosität der behandelten Menschen drastisch reduziert [21].

Die CD4-Zellzahl als Parameter für die Funktionsfähigkeit des Immunsystems beim HIV-positiven HCW kann nur beurteilt werden, wenn mehrere Werte über Monate vorliegen. Wenn die CD4-Zellzahl

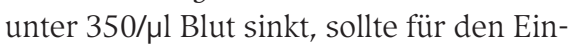
satz des HIV-positiven HCW auch das Risiko des Erwerbs einer nosokomialen/arbeitsbedingten Infektion (z. B. Tbc) berücksichtigt werden.

\section{Einsatz HIV-positiver HCW}

Die Festlegung von Grenzwerten, deren Unterschreitung ein nur geringes Transmissionsrisiko anzeigt, ist schwierig und kann sich nicht, wie bei nosokomialen HBV- und HCV-Fällen, an bekannten Übertragungsfällen orientieren. Vielmehr können hierfür lediglich Ergebnisse aus In-vitro- und tierexperimentellen Untersuchungen zugrunde gelegt werden (s. zuvor).

Grundsätzlich gelten für HIV-positive HCW klar definierte Auflagen, z. B. das Tragen doppelter Handschuhe, die mit steigender Viruslast ggf. modifiziert werden müssen. Daneben sind definierte Voraussetzungen zur Tätigkeitsausübung zu erfüllen (s. unten):

\section{Kernpunkte}

- Bei einer dauerhaften Viruslast von $\leq 50$ Kopien/ml können von dem HIV-positiven HCW alle operativen und invasiven Tätig- keiten durchgeführt werden.Wird im Rahmen der routinemäßigen Viruslastbestimmung eine HI-Viruslast zwischen 51 und $500 \mathrm{Kopien} / \mathrm{ml}$ ermittelt, ist abzuklären, ob es sich ggf. um einen „Blip“ handelt. "Blips ${ }^{\prime \prime}$ treten in der Regel nur kurzfristig $(<14$ Tage) auf und sind mit einer Viruslast $<500 \mathrm{Kopien/ml} \mathrm{assoziiert} \mathrm{[22].} \mathrm{Zur}$ Abklärung ist dann eine wiederholte Testung innerhalb von ca. 2 Wochen erforderlich. Ein HIV-positiver HCW, der zunächst eine konstante Viruslast $\leq 50 \mathrm{Ko}-$ pien/ml aufweist, bei dem jedoch kurzfristig (d. h. maximal ca. 14 Tage) Werte zwischen 51 und 500 Kopien/ml auftreten, kann weiterhin alle Tätigkeiten ausüben (ggf. unter individueller Berücksichtigung weiterer Parameter). Besteht die erhöhte Viruslast (51 bis 500 Kopien/ml) länger als ca. 14 Tage oder steigt die Viruslast auf $>500 \mathrm{Kopien} / \mathrm{ml}$, können nicht mehr alle operativen und invasiven Tätigkeiten ausgeübt und erst wieder aufgenommen werden, wenn die Viruslast konstant bei $\leq 50 \mathrm{Kopien} / \mathrm{ml}$ liegt.

- HCW mit einer HI-Viruslast von >50 Kopien/ml (länger als ca. 14 Tage) dürfen keine übertragungsträchtigen bzw. verletzungsträchtigen Operationen/Tätigkeiten durchführen. Nachfolgend werden unter Berücksichtigung der SHEA-Empfehlungen [6] und der DVV-Empfehlungen zu HBV und HCV [1] exemplarisch, ohne Anspruch auf Vollständigkeit, entsprechende Tätigkeiten aufgeführt:

- Beengtes Operationsfeld mit schlechter oder unterbrochener Sichtkontrolle (Operationen mit eingeschränktem Operationsfeld, beispielsweise bei oralchirurgischen oder abdominal- und thoraxchirurgischen Eingriffen in der Tiefe von Körperhöhlen, erfordern hohe Konzentration und technische Fertigkeiten des Operateurs und erhöhen die Verletzungswahrscheinlichkeit, insbesondere wenn manuelles Tasten bei gleichzeitiger Verwendung spitzer Instrumente erforderlich ist)

- Lange Operationsdauer (Handschuhwechsel alle 2-3 h erforderlich!) (Mit zunehmender Operationsdauer steigt die Anzahl okkulter Perforationen des Handschuhs und damit die Wahrscheinlichkeit einer Virustransmission. Nach einer Eingriffsdauer von 2-3 h sollten beide Handschuhpaare gewechselt werden)

- Mikroverletzungen durch Nahtmaterial [Kräftiges und häufiges Knoten birgt die Gefahr von Verletzungen insbesondere 
in den Beugefalten der Finger (kleine Schürfwunden bis hin zu tiefen Wunden) durch Einschneiden des Fadens in die Haut. Insbesondere geflochtenes Nahtmaterial kann als Depot oder Leitschiene für Virusmaterial zur Transmission führen]

- Manuelle Präparation in der Nähe von Instrumenten oder freistehenden Drähten oder Knochenfragmenten [Operationen mit manueller Führung und Tasten der Nadel sowie gleichzeitiges Arbeiten mit den Fingern und scharfen Instrumenten bergen ein hohes Risiko für Stich- oder Schnittverletzungen, welches sich im Falle von schwierig einsehbarem oder anatomisch bedingt schwer zugänglichem OP-Situs zusätzlich erhöht. Operationen mit spitzen Gegenständen im Gewebe (z. B. Verschluss der Sternotomie oder Anlegen von Cerclagen aus Draht/Stahl, scharfkantige Implantate oder spitze/scharfe Knochenfragmente) bergen mitunter das höchste Verletzungsrisiko. Ein erhöhtes Risiko für intraoperative Verletzungen geht in besonderem Maße vom Verschluss großer Operationszugänge aus, wenn die Wundränder manuell (meist vom Assistenten) und nicht von Instrumenten gehalten werden]

- Die HIV-Viruslast ist durch mindestens vierteljährliche Kontrollen zu überprüfen und die Einschätzung der Infektiosität nach dem jeweiligen Stand von Wissenschaft und Technik ggf. vorzunehmen. Der Sicherheitsspielraum der HI-Viruslast ist größer als bei der SHEA-Empfehlung [6] und so gewählt, dass auch vorübergehende Überschreitungen der HIV-Viruslast (bis $500 \mathrm{Kopien} / \mathrm{ml}$ ) angesichts von unvermeidbaren Messschwankungen toleriert werden können.

\section{Anmerkung}

Bei einer Erhöhung der Viruslast auf $>50$ Kopien/ml (länger als ca. 14 Tage)

- kann die Durchführung von Notfalleingriffen mit höchster Dringlichkeit bzw. die Ausweitung des Tätigkeitsfeldes auf verletzungsträchtige Notoperationen unter Berücksichtigung des Erfahrungs- und Ausbildungstandes des Mitarbeiters in Ausnahmefällen zugelassen werden. Dies sollte nur für diejenigen HCW gelten, die bereits einen hohen Kenntnisstand erworben haben und über große Erfahrung verfügen (z. B. Not-Sectio, Notfallthorakooder -laparotomie, Schockraumverant- wortung). Hier sind die Risiken einer Virustransmission gegen den Nutzen des Eingriffs kritisch abzuwägen;

-kann beispielsweise bei Großschadensereignissen, dringlichen Notfallindikationen oder lebensrettenden Soforteingriffen auf der Grundlage einer NutzenSchaden-Abwägung (Lebensrettung) grundsätzlich eine uneingeschränkte Tätigkeitsausübung gelten.

\section{Voraussetzungen für die Tätigkeitsausübung}

Die uneingeschränkte Tätigkeit des HIVpositiven HCW ist nur dann möglich, wenn Adhärenz bei der antiretroviralen Therapie besteht und wenn alle zur Vermeidung einer Infektionsübertragung erforderlichen Maßnahmen regelrecht eingehalten werden. Ferner ist ein vertrauensvolles Verhältnis zwischen Betriebsarzt und HCW hierbei Grundvoraussetzung.

Bei stabilen HI-Viruslastwerten $\leq 50 \mathrm{Ko}-$ pien/ml können alle operativen und invasiven Tätigkeiten durchgeführt werden, sofern die nachfolgend aufgeführten allgemeinen und verhaltenspräventiven Maßnahmen konsequent beachtet werden:

- Tragen doppelter Handschuhe bei invasiven und operativen Tätigkeiten,

- regelmäßige arbeitsmedizinische Betreuung,

- regelmäßige, mindestens vierteljährliche Kontrollen der HI-Viruslast,

- regelmäßige Betreuung durch einen in der HIV-Therapie erfahrenen Arzt,

- im Bedarfsfall Beratung durch eine Expertenkommission.

Zur Optimierung der Kontrolle der Adhärenz des betroffenen HCW ist die direkte Kommunikation zwischen dem Betriebsarzt und dem HIV-Behandler des HIV-positiven HCW dringend anzustreben.

\section{Postexpositionelle Prophylaxe}

Verletzt sich der HIV-positive HCW und es kommt zu einer Exposition von Patienten (bzw. Dritten, z. B. andere Mitarbeiter im $\mathrm{OP})$ mit dem Blut des HIV-positiven Mitarbeiters, sind die Betroffenen über das Risiko zu informieren, und ihnen ist unverzüglich eine Postexpositionsprophylaxe (PEP) anzubieten. Dies gilt auch, wenn ein begründeter Verdacht auf eine Exposition besteht, z. B. wenn nicht klar ist, ob aus einer Schnittverletzung des HIV-positiven HCW Blut in den OP-Situs gelangt ist.

Sollte es zur Übertragung größerer Mengen an Blut gekommen sein, muss - soweit möglich - unmittelbar eine PEP - auch in Narkose - erfolgen (mit Dokumentation und anschließender Aufklärung des Patienten).

Ferner ist es erforderlich, eine Blutprobe des HIV-positiven HCW zu asservieren, um - falls es tatsächlich zu einer HIV-Infektion des Exponierten kommt - den Ursprung dieser Infektion über eine Nukleinsäure-Sequenzanalyse abklären zu können, die Infektiosität (Viruslast) des Mitarbeiters zum Zeitpunkt der möglichen Transmission sowie evtl. vorhandene Resistenzen zu bestimmen. Ebenso ist eine Blutprobe des betroffenen Patienten zur Feststellung des Ausgangsstatus zu asservieren.

Grundsätzlich sind im Falle einer vermuteten akzidentiellen, nosokomialen HIVTransmission eine lückenlose und vertrauensvolle Kommunikation zwischen D-Arzt/ Betriebsarzt und Patient bzw. ggf. Dritten anzustreben und entsprechende Folgeuntersuchungen durchzuführen.

Die Wirksamkeit der medikamentösen HIV-Prophylaxe nach HIV-Exposition konnte bereits 1997 in einer retrospektiven Fallkontrollstudie gezeigt werden [23]. Nach einem Risikokontakt sollte der Beginn der PEP so schnell wie möglich, am besten innerhalb von 2 h erfolgen, da es bereits innerhalb weniger Stunden zur Anlagerung von HIV an die Wirtszelle und zur Integration des proviralen Genoms in die Zelle kommen kann. Nach mehr als $72 \mathrm{~h}$ wird in der Regel kein Prophylaxebeginn mehr empfohlen. Eine HIV-PEP sollte für die Dauer von 4 Wochen, in Einzelfällen auch länger, durchgeführt werden (s. auch PEPEmpfehlungen der Deutschen AIDS-Gesellschaft: http://www.daignet.de). Ist die Therapievorgeschichte der Indexperson bekannt, kann es aufgrund vorhandener Resistenzen gegen bestimmte HIV-Medikamente notwendig werden, in Zusammenarbeit mit einem erfahrenen Virologen/ HIV-Behandler die PEP entsprechend zu modifizieren.

Grundsätzlich ist jede Verletzung oder Schleimhautkontamination (z. B. von Mund, Auge oder Nase) mit Infektionsrisiko für einen Patienten oder Dritten an die zuständige Stelle (D-Arzt/Betriebsarzt) zu melden. Analog den DVV-Empfehlungen zu HBV 
und HCV [1] ist für den operativen Bereich ein Notfall- und Hygieneplan für die Vorgehensweise bei Verletzungen oder Schleimhautkontamination mit HIV-positivem Material zu erstellen. Der HIV-positive HCW ist über die Konsequenzen dieses Plans aufzuklären und muss diesem Plan zustimmen.

\section{Arbeitsmedizinische Aspekte}

Wie bei HBV- und/oder HCV-positiven HCW ist eine engmaschige arbeitsmedizinische Betreuung erforderlich, sodass einerseits gewährleistet ist, dass sich der HCW keiner Eigengefährdung aussetzt (z.B. durch eine nosokomiale/arbeitsbedingte Infektion) und andererseits im Falle von Veränderung des eigenen gesundheitlichen Zustandes (z. B. Begleiterkrankungen) der Einsatz des betroffenen Mitarbeiters $\mathrm{u}$. U. neu bewertet wird.

$\mathrm{Zu}$ berücksichtigen ist auch der Schutz des HIV-positiven HCW. Dazu gehört, dass empfohlene Impfungen für medizinisches Personal (z. B. Hepatitis A/B, MMR, VZV, Pertussis, Influenza) konsequent durchgeführt werden und dass bei signifikanter Reduktion der CD4-Zellzahl z. B. kein Einsatz in der Betreuung von Tbc-Erkrankten erfolgt.

Wichtige Grundlage für die Beurteilung des adäquaten Einsatzes eines HCW ist primär die vertrauensvolle Kommunikation zwischen ihm und seinem Betriebsarzt.

Beträgt die HI-Viruslast > 50 Kopien/ml (länger als ca. 14 Tage) ist in einem Gespräch zwischen dem HIV-positiven HCW und dem Betriebsarzt zu klären, ob eine Gefährdung Dritter vorliegt oder ob diese Gefährdung akut und/oder künftig ausgeschlossen werden kann (z. B. durch Vermeidung von Tätigkeiten mit Übertragungsgefahr bis die Viruslast wieder $\leq 50$ Kopien/ $\mathrm{ml}$ beträgt). Erfolgt diese Absprache (dokumentiert) einvernehmlich, sind keine weiteren Schritte erforderlich. In allen anderen Fällen ist die Hinzuziehung weiterer Personen (Expertengremium) notwendig. Die Zusammensetzung dieses Expertengremiums sowie der Ablauf der Vorgehensweise richten sich nach den Vorgaben des RKI und der DVV [1, 24].

\section{Ethische und juristische Aspekte}

Für medizinisches Personal besteht die Verantwortung gegenüber dem Patienten, dessen Gesundheitszustand nicht durch vermeidbare Infektionen, wie z. B. HBV, HCV, HIV etc., zu gefährden. Zu nennen ist hier das Prinzip der Nichtschädigung, die Fürsorgepflicht, die Anerkennung der Patientenselbstbestimmung, die informierte Zustimmung und die Pflichten, die aus der besonderen professionellen Beziehung zum Patienten erwachsen.

Unter Einhaltung der Vorgaben dieser Empfehlungen gehen von HIV-positiven HCW mit an Sicherheit grenzender Wahrscheinlichkeit keine Gefahren für Patienten aus, daher lässt sich aus ethischer Sicht argumentieren, dass keine Verpflichtung besteht, den Patienten über die Infektion des HCW zu informieren.

Kommt es trotz aller Vorsichtsmaßnahmen zu einer potenziellen Gefährdung eines Patienten, ist dieser über das Risiko zu informieren und ihm eine PEP anzubieten. Gleichzeitig ergibt sich die Forderung einer adäquaten Aufklärung zur PEP nach einem derartigen Zwischenfall. Die Schwierigkeit und Bedeutung dieser Krisenkommunikation werden häufig unterschätzt. Es ist aus ethischer und juristischer Sicht nicht nur erforderlich, dass der Patient informiert wird, sondern dass er diese Informationen auf umfassende und verständliche Weise erhält, um sich entscheiden zu können. Daher sollte die Information des Patienten beispielsweise durch einen in der HIV-Therapie erfahrenen Arzt unter Berücksichtigung der besonderen psychischen Situation erfolgen.

\section{HIV-positive HCW aus der Sicht der Aufsichtsbehörden}

Die HIV-Infektion ist nach Infektionsschutzgesetzes (IfSG) § 7 (3) lediglich nicht-namentlich an das Robert Koch-Institut (RKI) meldepflichtig, dennoch trifft den Mitarbeiter und die Betriebsleitung der jeweiligen Einrichtung im Gesundheitsdienst eine große Verantwortung, die insbesondere die behandelnden Patienten betrifft. Für Patienten muss in allen medizinischen Einrichtungen dafür Sorge getragen werden, dass die Wahrscheinlichkeit für Übertragungen jedweder Infektionserreger minimiert ist.

Im Sozialgesetzbuch werden Aussagen getroffen, die sich sowohl auf den Schutz der Beschäftigten als auch auf den Schutz der Patienten vor Gesundheitsgefahren beziehen (VII, § 21: „Der Unternehmer ist für die Durchführung der Maßnahmen zur Verhütung von arbeitsbedingten Gesundheitsgefahren verantwortlich. Die Versicherten haben nach ihren Möglichkeiten alle Maßnahmen zur Verhütung von [...] arbeitsbedingten Gesundheitsgefahren zu unterstützen und die entsprechenden Anweisungen des Unternehmers zu befolgen").

Auch bei „zufälliger ${ }^{\star \prime}$ Information sind die Gesundheitsämter gehalten, den Maßnahmenkatalog voll auszuschöpfen, da im IfSG nicht festgelegt wurde, dass dieses Wissen - unabhängig davon, wie es erhalten wurde - nicht verwertet werden darf (IfSG § 16: “(1) Werden Tatsachen festgestellt, die zum Auftreten einer übertragbaren Krankheit führen können, oder ist anzunehmen, dass solche Tatsachen vorliegen, so trifft die zuständige Behörde die notwendigen Maßnahmen zur Abwendung der dem Einzelnen oder der Allgemeinheit hierdurch drohenden Gefahren $[. . .]^{u \text { ) }}$.

Der HIV-positive HCW muss daher, sofern er in Bereichen arbeitet, die mit einem Übertragungsrisiko für Patienten einhergehen, Maßnahmen ergreifen, die die Ausbreitung jedweder Infektionserreger, nicht nur der HIV-Infektion, verhindern. Dies bedeutet, dass der HCW sich dem Betriebsarzt bzw. der Expertenkommission anvertrauen sollte. Der Betriebsarzt und die Expertenkommission unterliegen der Schweigepflicht bzw. tagen in unkritischen Fällen anonym. Es muss eine Gefährdungsanalyse des Arbeitsplatzes durchgeführt werden und ggf. die Weiterbeschäftigung des HIVpositiven HCW in nichtkritischen Bereichen erfolgen.

\section{Interessenkonflikt}

Der korrespondierende Autor gibt für sich und seine Koautoren an, dass kein Interessenkonflikt besteht.

\section{Literatur}

1. DVV 2007. Prävention der nosokomialen Übertragung von Hepatitis B Virus (HBV) und Hepatitis $C$ Virus (HCV) durch im Gesundheitswesen Tätige. http://www.dvv-ev.de/therapieempfehlungen/de/hbvhcv.pdf

2. Ciesielski C, Marianos D, Ou CY et al. Transmission of human immunodeficiency virus in a dental practice. Ann Intern Med 1992;116:798-805. 
3. Blanchard A, Ferris S, Chamaret S et al. Molecular evidence for nosocomial transmission of human immunodeficiency virus from a surgeon to one of his patients. J Virol 1998;72:4537-4540.

4. Goujon CP, Schneider VM, Grofti J et al. Phylogenetic analyses indicate an atypical nurse-topatient transmission of human immunodeficiency virus type 1. J Virol 2000;74:2525-2532.

5. Mallolas J, Arnedo M, Pumarola T et al. Transmission of HIV-1 from an obstetrician to a patient during a caesarean section. AIDS 2006; 20(2):285-287.

6. Henderson DK, Dembry L, Fishman NO et al. SHEA guideline for management of healthcare workers who are infected with hepatitis B virus, hepatitis $C$ virus, and/or human immunodeficiency virus. Infect Control Hosp Epidemiol 2010;31:203-232.

7. Gerberding JL, Rose DA, Ramiro NZ et al. Intraoperative provider injuries and potential patient recontacts at San Francisco General Hospital. Infect Control Hosp Epidemiol 1994;15:20.

8. Tokars JI, Bell DM, Culver DH et al. Percutaneous injuries during surgical procedures. JAMA 1992;267:2899-2904.

9. Gioananni P, Sinicco A, Cariti G et al. HIV infection acquired by a nurse. Eur J Epidemiol 1988;4:119-120.

10. Eberle J, Habermann J, Gürtler LG. HIV-1 infection transmitted by serum droplets into the eye: a case report. AIDS 2000;14:206-207.

11. Robert LM, Chamberland ME, Cleveland JL et al. Investigations of patients of health care workers infected with HIV. The Centers for Disease Control and Prevention Database. Ann Intern Med 1995;122:653-657.

12. Wicker S, Rabenau HF, Groneberg DA. Die HIV/ AIDS Epidemie und ihre Auswirkung auf die Arbeitswelt. Arbeitsmed Sozialmed Umweltmed 2010;45:64-68.

13. Statistisches Bundesamt 2011. http://www destatis.de

14. RKI. Schätzungen der Prävalenz und Inzidenz von HIV-Infektionen in Deutschland. Epidemio Bull 2011;46:415-425.

15. Internationales Arbeitsamt 2006. Ein praxisorientierter Leitfaden der IAO zu HIV/Aids in der Welt der Arbeit. http://www.ilo.org

16. Rusert P, Fischer M, Joos B et al. Quantification of infectious HIV-1 plasma viral load using a boosted in vitro infection protocol. Virology 2004;326:113-129.

17. Oksenhendler E, Harzic M, Le Roux JM et al HIV infection with seroconversion after a superficial needlestick injury to the finger. $\mathrm{N} \mathrm{Engl}$ J Med 1986;315:582.

18. Reid S, Juma OA. Minimum infective dose of HIV for parenteral dosimetry. Int J STD AIDS 2009;20:828-833.

19. Coll O, Hernandez M, Boucher CA et al. Vertical HIV-1 transmission correlates with a high maternal viral load at delivery. J Acquir Immune Defic Syndr Hum Retrovirol 1997;14:26-30.

20. Gulick RM. Antiretroviral treatment 2010: progress and controversies. J Acquir Immune Defic Syndr 2012;55(Suppl 1):43-48.

21. Cohen MS, Chen YQ, McCauley M et al. Prevention of HIV-1 infection with early antiretroviral therapy. N Engl J Med 2011;365:493-505.

22. Nettles RE, Kieffer TL, Kwon $P$ et al. Intermittent HIV-1 viremia (Blips) and drug resistance in patients receiving HAART. JAMA 2005;293(7):817-829.
23. Cardo DM, Culver DH, Ciesielski CA et al. A case-control study of HIV seroconversion in health care workers after percutaneous exposure. Centers for Disease Control and Prevention Needlestick Surveillance Group. N Engl J Med 1997;337:1485-1490.

24. Wicker S, Dickmann P, Rabenau HF et al. Etablierung einer risikoadaptierten Expertenkommission zur arbeitsmedizinischen Beurteilung von Mitarbeitern im Gesundheitswesen am Beispiel von HIV“. Arbeitsmed Sozialmed Umweltmed 2008;43:54-61.

Diese Empfehlung wurde von der DVV/GfV Kommission „HIV-Infektion bei medizinischem Personal“ erarbeitet. Der Kommission gehörten folgende Personen an:

Prof. Dr. Holger F. Rabenau (Vorsitzender)

- Virologie, Frau PD Dr. Dr. Sabine Wicker (stellvertretende Vorsitzende) - Arbeitsmedizin, Prof. Dr. Dr. René Gottschalk - ÖGD, Prof. Dr. Lutz Gürtler - Virologie, Frau Dr. Annette E. Haberl - Nationaler AIDS-Beirat/Deutsche AIDS-Gesellschaft, Dr. Osamah Hamouda Epidemiologie, Robert Koch-Institut (RKI), Dr. Heiko Himmelreich - Chirurgie, Notfallmedizin, Dr. Klaus Korn - Virologie, Nationales Referenzzentrum HIV, Prof. Dr. Thomas Mertens Virologie, GfV-Präsident, Dr. Kurt W. Schmidt - Ethiker, Dr. Stefan Schmiedel - Infektiologie, Prof. Dr. Andreas Spickhoff - Jurist, Frau Dipl. soz. päd. Gaby Wirz - AIDS-Hilfe, Prof. Dr. Peter Wutzler - Virologie - DVV-Präsident

\section{Hinweis}

Die vertretenen Positionen entsprechen der persönlichen Einstellung der Autoren und repräsentieren nicht zwangsläufig die Position der medizinischen Organisationen oder Institutionen, denen sie angehören. 